\title{
Proof of universality for the absorption of massive scalars by the higher-dimensional Reissner-Nordström black holes
}

\author{
Eylee Jung*, SungHoon $\mathrm{Kim}^{\dagger}$ and D. K. Park ${ }^{\ddagger}$, \\ Department of Physics, Kyungnam University, Masan, 631-701, Korea
}

\begin{abstract}
Motivated by black hole experiments as a consequence of the TeV-scale gravity arising from modern brane-world scenarios, we study the absorption problem for the massive scalars when the spacetime background is a $(4+n)$ dimensional Reissner-Nordström black hole. For analytic computation we adopt the near-extreme condition in the spacetime background. It is shown that the low-energy absorption cross section for the s-wave case holds an universality, i.e. the absorption cross section equals to the area of the black hole horizon divided by a velocity parameter.
\end{abstract}

\footnotetext{
*Email:eylee@kyungnam.ac.kr

†Email:shoon@kyungnam.ac.kr

‡Email:dkpark@hep.kyungnam.ac.kr
} 


\section{INTRODUCTION}

In the brane-world scenarios our four-dimensional universe is embedded in the higherdimensional bulk space. Although the higher-dimensional theories have their own long history [1], modern brane-world scenarios generally assume the large $[2,3]$ or warped $[4,5]$ extra dimensions. The most remarkable consequences arising from the modern brane-world scenarios seem to be a short-range behavior of the gravity deviated from the usual Newton law [5-12] and the emergence of the Tev-scale gravity. Especially, the emergence of the Tev-scale gravity provides a motivation [13-15] for the black hole experiments in the future accelerator such as the CERN Large Hadron Collider. Thus, it is important to investigate the effect of the extra dimensions in the various properties of black holes.

In this context, recently, the absorption and emission problems for the $(4+n)$-dimensional Schwarzschild black hole are examined [16,17]. Especially, Ref. [17] shows that the ratio factor $1 / 8$ [18] of the low-energy absorption cross sections for the massive scalar and Dirac fermion minimally coupled to the four-dimensional Schwarzschild black hole is changed into $2^{-(n+3) /(n+1)}$ when we have $n$-dimensional toroidally compactified extra space.

In this letter we would like to examine the absorption problem of the massive scalar minimally coupled to the $(4+n)$-dimensional Reissner-Nordström(RN) black hole. In particular we will show that the low-energy absorption cross section for the s-wave massive scalar holds the generalized universality [17], i.e. $\sigma \sim \mathcal{A}_{h} / \bar{v}$, where $\mathcal{A}_{h}$ is an area of the horizon hyper-

surface and $\bar{v}=\sqrt{1-m^{2} / \omega^{2}}$ is a velocity parameter introduced firstly in Ref. [18]. In fact, this formula is a generalization of the universality for the massless scalar examined in Ref. $[19]$.

\section{SOULTIONS}

The $(4+n)$-dimensional RN spacetime is well-known [20,21] whose metric is explicitly

$$
d s^{2}=-\left[1-\left(\frac{\tilde{r}_{+}}{\tilde{r}}\right)^{n+1}\right]\left[1-\left(\frac{\tilde{r}_{-}}{\tilde{r}}\right)^{n+1}\right] d t^{2}+\frac{d \tilde{r}^{2}}{\left[1-\left(\frac{\tilde{r}_{+}}{\tilde{r}}\right)^{n+1}\right]\left[1-\left(\frac{\tilde{r}_{-}}{\tilde{r}}\right)^{n+1}\right]}+\tilde{r}^{2} d \Omega_{n+2}^{2}
$$


where $\tilde{r}_{ \pm}$represent the outer and inner horizons. Thus, $\tilde{r}_{-}=0$ and $\tilde{r}_{-}=\tilde{r}_{+}$means the Schwarzschild and extremal limits, respectively. The mass $M$ and charge $Q$ of the RN black hole are given by

$$
\begin{aligned}
& M=\frac{n+2}{16 \pi G} \Omega_{n+2}\left(\tilde{r}_{+}^{n+1}+\tilde{r}_{-}^{n+1}\right) \\
& Q= \pm\left(\tilde{r}_{+} \tilde{r}_{-}\right)^{\frac{n+1}{2}} \sqrt{\frac{(n+1)(n+2)}{8 \pi G}}
\end{aligned}
$$

where $\Omega_{n+2}$ is the area of a unit $(n+2)$-sphere

$$
\Omega_{n+2}=\frac{2 \pi^{\frac{n+3}{2}}}{\Gamma\left(\frac{n+3}{2}\right)}
$$

For computation of the absorption cross section it is convenient to introduce the new parameters $r_{0}$ and $r_{1}$ as following:

$$
\tilde{r}_{+}=\left(r_{1}^{n+1}+r_{0}^{n+1}\right)^{\frac{1}{n+1}} \quad \tilde{r}_{-}=r_{1}
$$

Then the spacetime metric (1) reduces to

$$
d s^{2}=-h(r) f^{-n-1}(r) d t^{2}+f(r)\left[h^{-1}(r) d r^{2}+r^{2} d \Omega_{n+2}^{2}\right]
$$

where $r$ is a new radial coordinate defined

$$
\tilde{r}^{2}=f(r) r^{2}
$$

and

$$
f(r)=\left[1+\left(\frac{r_{1}}{r}\right)^{n+1}\right]^{\frac{2}{n+1}} \quad h(r)=1-\left(\frac{r_{0}}{r}\right)^{n+1} .
$$

Of course, $d \Omega_{n+2}^{2}$ is an angle-dependent part of the metric

$$
d \Omega_{n+2}^{2}=d \theta_{1}^{2}+\sin ^{2} \theta_{1}\left[d \theta_{2}^{2}+\sin ^{2} \theta_{2}\left\{d \theta_{3}^{2}+\cdots+\sin ^{2} \theta_{n}\left(d \theta_{n+1}^{2}+\sin ^{2} \theta_{n+1} d \phi^{2}\right) \cdots\right\}\right] .
$$

If $n=1$, the metric (5) exactly coincides with a five-dimensional solution of the low-energy action of type-IIB string theory compactified on a torus explicitly introduced in Ref. [22] on condition that three charges $Q_{1}, Q_{5}$ and $Q_{K}$ in Ref. [22] are all same. In this letter, however, 
we will consider the arbitrary number of extra dimensions. Also we omit the configuration of the electromagnetic field, which is not necessary throughout the paper.

Now, we consider a scalar field $\Phi$ minimally coupled to the spacetime (5). Then the usual wave equation $\left(\square-m^{2}\right) \Phi=0$ reduces to the following radial equation:

$$
\left[\frac{h}{r^{n+2}} \partial_{r}\left(r^{n+2} h \partial_{r}\right)-\frac{\ell(\ell+n+1)}{r^{2}} h-m^{2} f h+f^{n+2} \omega^{2}\right] R=0
$$

where we assume the separability $\Phi=e^{-i \omega t} R(r) \tilde{Y}_{\ell}(\Omega)$ and $\tilde{Y}_{\ell}$ is an higher-dimensional spherical harmonics. What we want to do is to solve Eq.(9) in the asymptotic and nearhorizon regimes separately. Matching one solution with another, we would like to extract information on the greybody factor and the absorption cross section.

In the asymptotic region $r \sim \infty$, we have $h \sim f \sim 1$ which makes Eq.(9) simply

$$
\left[\partial_{r}^{2}+\frac{n+2}{r} \partial_{r}+\omega^{2} \bar{v}^{2}\right] R_{F F}^{s}=0
$$

where $\bar{v}=1-m^{2} / \omega^{2}$ and we confined our attention to the $s$-wave, i.e. $\ell=0$. For the case of non-zero angular momentum, i.e. $\ell \neq 0$, it seems to be very difficult to solve Eq.(9) even in the asymptotic region. Also the solution should be non-trivially dependent on the number of extra dimensions in this case. Since our main interest is focused on the universality of the low-energy absorption cross section, we choose $\ell=0$ for simplicity. The subscript of $R$ in Eq.(10) stands for "far field" and the superscript indicates our restriction to the $s$-wave. The solution of Eq.(10) is easily expressed in terms of the Bessel functions:

$$
R_{F F}^{s}=r^{-\frac{n+1}{2}}\left[A_{1} J_{\frac{n+1}{2}}(\omega \bar{v} r)+A_{2} Y_{\frac{n+1}{2}}(\omega \bar{v} r)\right]
$$

Next we would like to solve the radial equation (9) in the near-horizon region $r \sim r_{0}$, which corresponds to $\tilde{r} \sim \tilde{r}_{+}$. It is convenient for further analysis to introduce a new variable $v=\left(r_{0} / r\right)^{n+1}$, which changes Eq.(9) into

$$
\left[(1-v) \frac{d}{d v}(1-v) \frac{d}{d v}-\frac{\ell(\ell+n+1)(1-v)}{(n+1)^{2} v^{2}}-\frac{m^{2}(1-v) f}{(n+1)^{2} r_{0}^{n+1} v} r^{n+3}+\frac{\omega^{2} f^{n+2}}{(n+1)^{2} r_{0}^{n+1} v} r^{n+3}\right] R=0 .
$$


It seems to be formidable to solve Eq.(12) in the near-horizon region $v \sim 1$. Thus, we restrict our attention to the near-extremal case, i.e. $r_{0} \sim 0$, for analytical analysis. With this restriction $f(r)$ can be expanded as a power series with an expansion parameter $r_{0} / r_{1}$. Then the radial equation (12) reduces to

$$
\left[(1-v) \frac{d}{d v}(1-v) \frac{d}{d v}+\left(D+\frac{C}{v}+\frac{G}{v^{2}}\right)\right] R^{N E}=0
$$

where the superscript $N E$ indicates the near-extremal case and

$$
\begin{aligned}
& D=\frac{\omega^{2} r_{0}^{2}}{(n+1)^{2}}\left(\frac{r_{1}}{r_{0}}\right)^{2 n+4} \\
& C=\frac{m^{2} r_{1}^{2}}{(n+1)^{2}}+\frac{2(n+2) \omega^{2} r_{0}^{2}}{(n+1)^{3}}\left(\frac{r_{1}}{r_{0}}\right)^{n+3}+\frac{\ell(\ell+n+1)}{(n+1)^{2}} \\
& G=-\frac{m^{2} r_{1}^{2}}{(n+1)^{2}}\left[1-\frac{2}{n+1}\left(\frac{r_{0}}{r_{1}}\right)^{n+1}\right]+\frac{(n+2)(n+3) \omega^{2} r_{1}^{2}}{(n+1)^{4}}-\frac{\ell(\ell+n+1)}{(n+1)^{2}} .
\end{aligned}
$$

In order to solve Eq.(13) in the near-horizon region, we introduce $z=1-v$ and $R^{N E}=$ $z^{-i(a+b) / 2} F^{N E}$, where the constants $a$ and $b$ will be fixed later. Then Eq.(13) becomes

$$
\begin{gathered}
z(1-z) \frac{d^{2} F^{N E}}{d z^{2}}+(1-i a-i b)(1-z) \frac{d F^{N E}}{d z} \\
+\left[\left(\frac{(a+b)^{2}}{4}-D\right)+\frac{1}{z}\left(D+C+G-\frac{(a+b)^{2}}{4}\right)+\frac{G}{1-z}\right] F^{N E}=0 .
\end{gathered}
$$

Since $z \sim 0$ in the near-horizon region, we can simplify Eq.(15) in this region as

$$
\begin{gathered}
z(1-z) \frac{d^{2} F_{N H}^{N E}}{d z^{2}}+(1-i a-i b)(1-z) \frac{d F_{N H}^{N E}}{d z} \\
+\left[\left(\frac{(a+b)^{2}}{4}-D+G\right)+\frac{1}{z}\left(D+C+G-\frac{(a+b)^{2}}{4}\right)\right] F_{N H}^{N E}=0
\end{gathered}
$$

where the subscript $N H$ denotes the near-horizon. Choosing

$$
\begin{aligned}
& a=\sqrt{D+C+G}+\sqrt{D-G} \\
& b=\sqrt{D+C+G}-\sqrt{D-G},
\end{aligned}
$$

we can solve Eq.(16) in terms of the hypergeometric functions. Thus, $R^{N E}$ in the nearhorizon region, say $R_{N H}^{N E}$, becomes

$$
R_{N H}^{N E}=\alpha_{I} z^{-i \frac{a+b}{2}} F(-i a,-i b ; 1-i a-i b ; z)+\alpha_{I I} z^{i \frac{a+b}{2}} F(i a, i b ; 1+i a+i b ; z) .
$$


Since the scalar wave should be purely ingoing in this region due to the physically relevant reason, we should choose $\alpha_{I I}=0$, which yields

$$
R_{N H}^{N E}=\alpha_{I} z^{-i \frac{a+b}{2}} F(-i a,-i b ; 1-i a-i b ; z) .
$$

If we take $z \rightarrow 0$ limit in $R_{N H}^{N E}$, it is easy to show

$$
R_{N H}^{N E} \sim \alpha_{I} z^{-i \frac{a+b}{2}}=\alpha_{I} e^{-i \sqrt{D+C+G} \ln (1-v)}=\alpha_{I} e^{-\frac{i}{2}(a+b) \ln (1-v)}
$$

as expected.

\section{COMPUTATION OF ABSORPTION QUANTITIES}

Now, we would like to compute the quantities related to the absorption via the matching between the near-horizon solution (20) and the asymptotic solution (11). If we take $z \rightarrow 1$ limit in Eq.(19), it is easy to show

$$
\begin{aligned}
\lim _{z \rightarrow 1} R_{N H}^{N E}=\alpha_{I} z^{-i \frac{a+b}{2}}[ & \frac{\Gamma(1-i a-i b)}{\Gamma(1-i a) \Gamma(1-i b)} F(-i a,-i b ; \epsilon ; v) \\
& \left.+v \frac{\Gamma(1-i a-i b) \Gamma(-1+\epsilon)}{\Gamma(-i a) \Gamma(-i b)} F(1-i a, 1-i b ; 2 ; v)\right]
\end{aligned}
$$

where the regularization parameter $\epsilon=0^{+}$is introduced. Since $v \sim 0$ when $z \sim 1$, one can expand Eq.(21) as a power series of $v$. Then the contribution to the linear term from the first term in Eq.(21) involves an infinity proportional to $1 / \epsilon$. This factor, however, is exactly cancelled out by the contribution from the second term in Eq.(21). Thus, expanding Eq.(21) simply yields

$$
\lim _{r \rightarrow \infty} R_{N H}^{N E}=\alpha_{I}\left[\mathcal{E}+\mathcal{H}\left(\frac{r_{0}}{r}\right)^{n+1}\right]
$$

where

$$
\mathcal{E}=\frac{\Gamma(1-i a-i b)}{\Gamma(1-i a) \Gamma(1-i b)}
$$

and $\mathcal{H}$ is a constant whose explicit expression is not needed. 
Next, we take $r \rightarrow 0$ limit in Eq.(11), which is easily carried out by making use of the series expansions of the Bessel functions:

$$
\lim _{r \rightarrow 0} R_{F F}^{s}=A_{1}\left[\frac{\left(\frac{\omega \bar{v}}{2}\right)^{\frac{n+1}{2}}}{\Gamma\left(\frac{n+3}{2}\right)}+\cdots\right]+A_{2}\left[-\frac{\Gamma\left(\frac{n+1}{2}\right)\left(\frac{2}{\omega \bar{v}}\right)^{\frac{n+1}{2}}}{\pi} \frac{1}{r^{n+1}}+\cdots\right] .
$$

Comparing Eq.(22) with Eq.(24) straightforwardly leads

$$
\begin{aligned}
& A_{1}=\alpha_{I} \frac{\Gamma\left(\frac{n+3}{2}\right)}{\left(\frac{\omega \bar{v}}{2}\right)^{\frac{n+1}{2}}} \mathcal{E} \\
& A_{2}=-\pi \alpha_{I} \frac{r_{0}^{n+1}}{\Gamma\left(\frac{n+1}{2}\right)\left(\frac{2}{\omega \bar{v}}\right)^{\frac{n+1}{2}}} \mathcal{H} .
\end{aligned}
$$

It is worthwhile noting $A_{1}>>A_{2}$ in the low-energy approximation, i.e. $\omega<<1$, which will be used later.

In order to compute the greybody factor we take $r \rightarrow \infty$ limit in $R_{F F}^{s}$ :

$$
\lim _{r \rightarrow \infty} R_{F F}^{s}=\varphi_{i n}+\varphi_{r e}
$$

where $\varphi_{\text {in }}$ and $\varphi_{\text {re }}$ are respectively the incident and reflected waves, whose explicit forms are

$$
\begin{aligned}
\varphi_{i n} & =\frac{A_{1}+i A_{2}}{\sqrt{2 \pi \omega \bar{v} r^{n+2}}} e^{-i\left[\omega \bar{v} r-\frac{n+2}{4} \pi\right]} \\
\varphi_{r e} & =\frac{A_{1}-i A_{2}}{\sqrt{2 \pi \omega \bar{v} r^{n+2}}} e^{i\left[\omega \bar{v} r-\frac{n+2}{4} \pi\right]} .
\end{aligned}
$$

From a definition of the conserved flux

$$
j \equiv \frac{1}{2 i}\left(\Psi^{*} h r^{n+2} \frac{d \Psi}{d r}-\text { c.c. }\right)
$$

the ingoing flux is exactly calculated:

$$
j_{i n}=\frac{1}{2 i}\left(\phi_{i n}^{*} h r^{n+2} \frac{d \phi_{i n}}{d r}-\text { c.c. }\right)=-\frac{1}{2 \pi}\left|A_{1}+i A_{2}\right|^{2} .
$$

By the same way it is straightforward to compute the transmitted flux using Eq.(20):

$$
j_{t r}=-\frac{n+1}{2}(a+b) r_{0}^{n+1}\left|\alpha_{I}\right|^{2} .
$$


Thus the greybody factor $\mathcal{F}$ becomes

$$
\mathcal{F} \equiv\left|\frac{j_{t r}}{j_{\text {in }}}\right|=(n+1) \pi(a+b) r_{0}^{n+1}\left|\frac{\alpha_{I}}{A_{1}+A_{2}}\right|^{2} \approx(n+1) \pi(a+b) r_{0}^{n+1}\left|\frac{\alpha_{I}}{A_{1}}\right|^{2},
$$

where the last approximation comes from $\left|A_{1}\right|>>\left|A_{2}\right|$ in the low-energy approximation. Combining Eq.(25) and (31), therefore, yields

$$
\mathcal{F}=(n+1) \pi(a+b) r_{0}^{n+1} \frac{\left(\frac{\omega \bar{v}}{2}\right)^{n+1}}{\Gamma^{2}\left(\frac{n+3}{2}\right)}\left|\frac{\Gamma(1-i a) \Gamma(1-i b)}{\Gamma(1-i a-i b)}\right|^{2} .
$$

Using a property of the gamma function $|\Gamma(1-i x)|^{2}=\pi x / \sinh x$, it is straightforward to re-express Eq.(32) in the form

$$
\mathcal{F}=2(n+1) \pi^{2} a b \frac{\left(\frac{\omega \bar{v} r_{0}}{2}\right)^{n+1}}{\Gamma^{2}\left(\frac{n+3}{2}\right)} \frac{e^{2 \pi(a+b)}-1}{\left(e^{2 \pi a}-1\right)\left(e^{2 \pi b}-1\right)} .
$$

Since $D>C>C$ which is easily deduced from Eq.(14) with a condition of the near-extremal limit, Eq.(17) indicates

$$
\begin{aligned}
& a \sim 2 \sqrt{D}=2 \frac{\omega r_{0}}{n+1}\left(\frac{r_{1}}{r_{0}}\right)^{n+2} \propto r_{0}^{-n-1} \\
& b \sim \frac{C}{2 \sqrt{D}} \sim \frac{(n+2) \omega r_{1}}{(n+1)^{2}} \propto r_{0}^{0}
\end{aligned}
$$

where we fix $\ell=0$ in Eq.(14) for the restriction to the $s$-wave. Eq.(34) also indicates $a>>b$. With this approximation, therefore, the greybody factor (33) reduces to

$$
\mathcal{F} \approx 2 \pi r_{1}^{n+2} \omega \frac{\left(\frac{\omega \bar{v}}{2}\right)^{n+1}}{\Gamma^{2}\left(\frac{n+3}{2}\right)}
$$

The relation between the absorption cross section and the greybody factor in the higher dimensions can be derived using a $(4+n)$-dimensional optical theorem [23]

$$
\sigma_{\ell}(\omega)=\frac{2^{n} \Gamma\left(\frac{n+3}{2}\right)}{\pi\left(\omega \bar{v} r_{H}\right)^{n+2}} \mathcal{A}_{h} \frac{(2 \ell+n+1)(\ell+n) !}{(n+1) ! \ell !} \mathcal{F}
$$

where $r_{H}$ and $\mathcal{A}_{h}$ are respectively the horizon radius and the area of the horizon hypersurface. For the s-wave case Eq.(36) implies

$$
\sigma_{s}(\omega)=\frac{2^{n} \Gamma^{2}\left(\frac{n+3}{2}\right)}{\pi\left(\omega \bar{v} r_{1}\right)^{n+2}} \mathcal{A}_{h} \mathcal{F}=\frac{\mathcal{A}_{h}}{\bar{v}}
$$


where $\mathcal{A}_{h}$ is for our case

$$
\mathcal{A}_{h}=\frac{2 \pi^{\frac{n+3}{2}}}{\Gamma\left(\frac{n+3}{2}\right)} r_{1}^{n+2} .
$$

Thus the universality for the low-energy absorption cross section is valid in the higherdimensional RN as well as Schwarzschild black holes.

\section{CONCLUSION}

In this letter we have examined the absorption problem for the massive scalars by the higher-dimensional RN black holes. For analytic computation we have chosen the nearextreme condition of the RN black holes. However, it seems to be greatly nice if we can remove this near-extreme condition. If it is possible, we may be able to study the absorption and emission problems in the Schwarzschild and RN black holes as an unified way. Apparently, it is beyond the dilute gas region [24]. To relax the near-extremal condition we should solve Eq.(12) in the near-horizon region without relying on $r_{0} \sim 0$, which seems to be an highly non-trivial problem. Although it is assumed to be possible to solve it approximately, we should check whether this solution gives physically reasonable results through a matching with the asymptotic solution. This should be another difficult problem. Perhaps, numerical approach can be a breakthrough. For this we may use the numerical method of Ref. [25] from the beginning. It may be possible in this approach to relax the s-wave restriction too. We hope to consider this elsewhere.

Acknowledgement: This work was supported by the Korea Research Foundation Grant (KRF-2003-015-C00109). 


\section{REFERENCES}

[1] H. C. Lee (Ed.), An introduction to Kaluza-Klein Theories (World Scientific, Singapore, 1984).

[2] N. Arkani-Hamed, S. Dimopoulos and G. Dvali, Phys. Lett. B429 (1998) 263 [hep$\mathrm{ph} / 9803315]$.

[3] L. Antoniadis, N. Arkani-Hamed, S. Dimopoulos and G. Dvali, Phys. Lett. B436 (1998) 257 [hep-ph/9804398].

[4] L. Randall and R. Sundrum, Phys. Rev. Lett. 83 (1999) 3370 [hep-ph/9905221].

[5] L. Randall and R. Sundrum, Phys. Rev. Lett. 83 (1999) 4690 [hep-th/9906064].

[6] J. Garriga and T. Tanaka, Phys. Rev. Lett. 84 (2000) 2778 [hep-th/9911055].

[7] S. B. Giddings, E. Katz and L. Randall, JHEP 0003 (2000) 023 [hep-th/0002091].

[8] M. J. Duff and J. T. Liu, Phys. Rev. Lett. 85 (2000) 2052 [hep-th/0003237].

[9] G. Dvali, G. Gabadadze and M. Porrati, Phys. Lett. B 485 (2000) 208 [hep-th/0005016].

[10] G. Dvali, G. Gabadadze, M. Kolanović and F. Nitti, Phys. Rev. D 64 (2001) 084004 [hep-ph/0102216].

[11] E. Jung, S. H. Kim and D. K. Park, Nucl. Phys. B669 (2003) 306 [hep-th/0305156].

[12] D. K. Park, S. Tamaryan and Y. G. Miao, Phys. Lett. B 578 (2004) 443 [hepth/0309071].

[13] S. B. Giddings and T. Thomas, Phys. Rev. D65 (2002) 056010 [hep-ph/0106219].

[14] S. Dimopoulos and G. Landsberg, Phys. Rev. Lett. 87 (2001) 161602 [hep-ph/0106295].

[15] D. M. Eardley and S. B. Giddings, Phys. Rev. D66 (2002) 044011 [gr-qc/0201034].

[16] C. M. Harris and P. Kanti, JHEP 0310 (2003) 014 [hep-ph/0309054]. 
[17] E. Jung, S. H. Kim and D. K. Park, will appear in JHEP [hep-th/0406117].

[18] W. G. Unruh, Phys. Rev. D14 (1976) 3251.

[19] S. R. Das, G. Gibbons, and S. D. Mathur, Phys. Rev. Lett. 78 (1997) 417 [hepth/9609052].

[20] F. R. Tangherlini, Nuovo Cimento 27 (1963) 636.

[21] R. C. Myers and M. J. Perry, Ann. Phys. 172 (1986) 304.

[22] S. W. Hawking and M. M. Taylor-Robinson, Phys. Rev. D55 (1997) 7680 [hepth/9702045].

[23] S. S. Gubser, Phys. Rev. D56 (1997) 4984 [hep-th/9704195].

[24] J. Maldacena and A. Strominger, Phys. Rev. D 55 (1997) 861 [hep-th/9609026].

[25] E. Jung and D. K. Park, Class. Quant. Grav. 21 (2004) 3717 [hep-th/0403251]. 